\title{
Evidence for a pressure-induced spin transition in olivine-type $\mathrm{LiFePO}_{4}$ triphylite
}

\author{
Maribel Núñez Valdez, ${ }^{1, *}$ Ilias Efthimiopoulos, ${ }^{1,2, \dagger}$ Michail Taran, ${ }^{1,3}$ Jan Müller, ${ }^{1,4}$ Elena Bykova, ${ }^{5}$ Catherine McCammon, ${ }^{6}$ \\ Monika Koch-Müller, ${ }^{1}$ and Max Wilke ${ }^{2}$ \\ ${ }^{1}$ GFZ German Research Centre for Geosciences, Telegrafenberg, 14473 Potsdam, Germany \\ ${ }^{2}$ Institute of Earth and Environmental Science, University of Potsdam, Karl-Liebknecht-Strasse 24-25, 14476 Potsdam-Golm, Germany \\ ${ }^{3}$ National Academy of Sciences of Ukraine, Institute of Geochemical Mineral and Ore Format, UA-03680 Kiev 142, Ukraine \\ ${ }^{4}$ Institute of Geology and Mineralogy, University of Cologne, Zulpicher Strasse 49b, 50674 Cologne, Germany \\ ${ }^{5}$ FS-PE, PETRA III, Deutsches Elektronen Synchrotron, 22607 Hamburg, Germany \\ ${ }^{6}$ Bayerisches Geoinstitut, Universität Bayreuth, 95440 Bayreuth, Germany
}

(Received 17 January 2018; revised manuscript received 13 April 2018; published 3 May 2018)

\begin{abstract}
We present a combination of first-principles and experimental results regarding the structural and magnetic properties of olivine-type $\mathrm{LiFePO}_{4}$ under pressure. Our investigations indicate that the starting $\mathrm{Pbnm}$ phase of $\mathrm{LiFePO}_{4}$ persists up to $70 \mathrm{GPa}$. Further compression leads to an isostructural transition in the pressure range of 70-75 GPa, inconsistent with a former theoretical study. Considering our first-principles prediction for a high-spin to low-spin transition of $\mathrm{Fe}^{2+}$ close to $72 \mathrm{GPa}$, we attribute the experimentally observed isostructural transition to a change in the spin state of $\mathrm{Fe}^{2+}$ in $\mathrm{LiFePO}_{4}$. Compared to relevant Fe-bearing minerals, $\mathrm{LiFePO}_{4}$ exhibits the largest onset pressure for a pressure-induced spin state transition.
\end{abstract}

DOI: 10.1103/PhysRevB.97.184405

\section{INTRODUCTION}

Compounds belonging to the lithiophilite-triphylite series $\mathrm{Li} M 2 \mathrm{PO}_{4}$ where $M 2$ represents a metal $(M 2=\mathrm{Mn}, \mathrm{Fe})$ are commonly found throughout the Earth's crust and mantle in pegmatites with high $\mathrm{Li}$ and $\mathrm{P}$ content $[1,2]$. At ambient conditions, they are isostructural with $(\mathrm{Mg}, \mathrm{Fe})_{2} \mathrm{SiO}_{4}$-olivine [space-group (SG) 62, Pbnm and $Z=4$ ], one of the most abundant minerals $(\sim 50-60 \% \mathrm{vol})$ in the upper mantle of our planet $(<410 \mathrm{~km}$ depth and pressure of $P<13.5 \mathrm{GPa})$ [3]. Figure 1 shows this olivine-type structure, which can be seen as a distorted hexagonal close packing of oxygen anions with corner-sharing $M 2 \mathrm{O}_{6}$ and edge-sharing $\mathrm{LiO}_{6}$ octahedra aligned in parallel chains along the $\mathbf{b}$ axis. The $\mathrm{P}$ ions are in $\mathrm{PO}_{4}$ tetrahedra linking the octahedral layers.

The transition-metal $M 2$ in these $\mathrm{Li} M 2\left(\mathrm{PO}_{4}\right)$ phosphate materials is octahedrally coordinated (Fig. 1), which leads to the crystal-field splitting ( $\Delta$ ) of the $M 23 d$ electrons into three lower-energy levels $t_{2 g}$ and two higher-energy levels $e_{g}$. At low pressures, Hund's rule predicts that, for example, $M 2=\mathrm{Fe}^{2+}$ (ferrous iron) adopts the high-spin (HS) state with $S=2\left(t_{2 g}^{4} e_{g}^{2}\right)$. However, as pressure increases, $\Delta$ increases at the expense of the spin-pairing exchange energy $J(J<$ $\Delta$ ), and $\mathrm{Fe}^{2+}$ undergoes a spin transition to the energetically more favorable low-spin (LS) state with $S=0\left(t_{2 g}^{6} e_{g}^{0}\right)$. Using computational and experimental (EXP) approaches, this pressure-induced spin crossover has been investigated and reported in several Fe-bearing minerals of the Earth's lower mantle (in the range of 660-2890-km depth and 23-135 GPa), such

\footnotetext{
*mari_nv@gfz-potsdam.de

†iliefthi@gfz-potsdam.de
}

as $(\mathrm{Mg}, \mathrm{Fe}) \mathrm{O}$-ferropericlase [4-17], $(\mathrm{Mg}, \mathrm{Fe}) \mathrm{SiO}_{3}$-bridgmanite [18-25], and $(\mathrm{Mg}, \mathrm{Fe}) \mathrm{CO}_{3}$-magnesiosiderite [26-36]. The study of this HS-to-LS transition is important as it has effects on the structural, optical, elastic, and thermodynamic properties of the Fe-bearing host minerals and in consequence potential and profound geophysical repercussions in the interpretation of seismic velocities and geodynamical modeling [12,16,37]. In addition to the aforementioned minerals, it was reported using density functional theory (DFT) $[39,40]$ that $\mathrm{LiFePO}_{4}$-triphylite also undergoes a HS-to-LS spin crossover at about $52 \mathrm{GPa}$ although not in the Pbnm structure but rather as a $\mathrm{Cmcm}$ high-pressure (HP) and hightemperature (HT) phase, which was shown to become the ground state at $\sim 4 \mathrm{GPa}$ [41]. Experimentally, the Pbnm $\rightarrow$ $\mathrm{Cmcm}$ structural transformation was observed at $6.5 \mathrm{GPa}$ and $1173 \mathrm{~K}$ [42]. Recently, however, some controversy regarding the validity of the structural and spin transitions has emerged as recent experimental and computational studies addressing the structural stability of $\mathrm{LiFePO}_{4}$ under pressure $[43,44]$ have shown that the Pbnm phase is stable up to $\sim 30 \mathrm{GPa}$. Thus, leaving somewhat open and unresolved the issues of whether or not: (1) the Pbnm $\rightarrow \mathrm{Cmcm}$ structural phase transition occurs in triphylite at room temperature (RT), and if not, (2) does the Pbnm phase undergo a HS-to-LS transition similar to other Fe-bearing minerals $[4,18,26]$ ?

Aside from the geophysical relevance of these $\mathrm{Li} M 2 \mathrm{PO}_{4}$ olivine-type materials, in recent years, $\mathrm{LiFePO}_{4}$-triphylite has also attracted considerable interest as a storage cathode material in rechargeable Li batteries (for a review see, e.g., Ref. [45] and references therein). For example, $\mathrm{Li}^{+}$migration depends on the compressibility and structural symmetry of $\mathrm{LiFePO}_{4}$-triphylite [44]. Hence, a thorough study of triphylite will allow us to better understand the properties of this material and its potential high-pressure applications. 


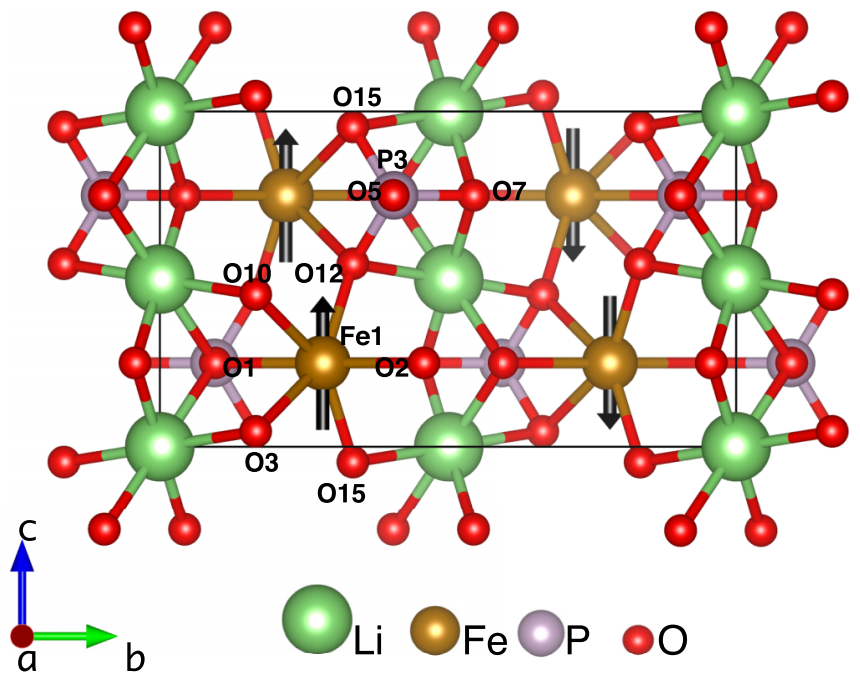

(a)

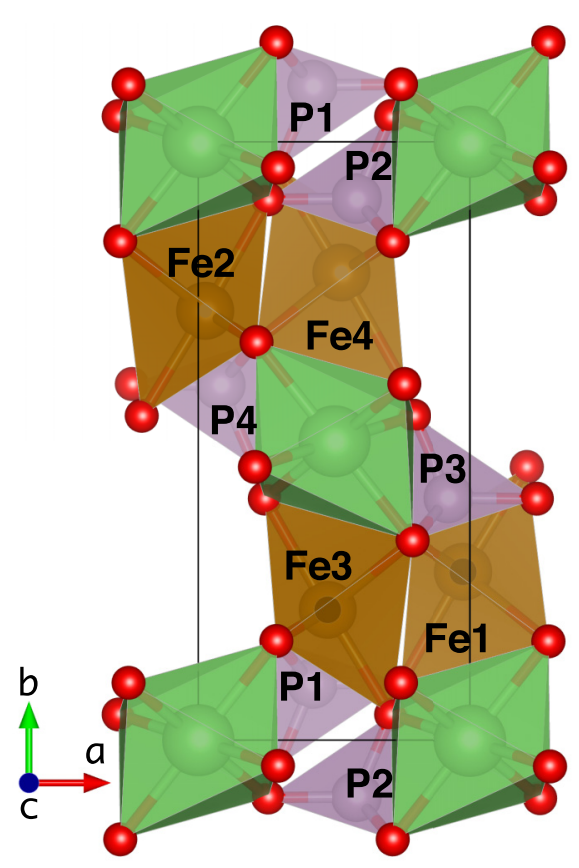

(b)

FIG. 1. At ambient pressure, $\mathrm{LiFePO}_{4}$-triphylite $(M 2=\mathrm{Fe})$ crystallizes in the olivine-type structure (SG 62, Pbnm, Z=4) with antiferromagnetic (AFM) ordering. Selected Fe, P, and $\mathrm{O}$ atoms are indicated for later analysis in Sec. IV. (Crystal structures in this paper are visualized using VESTA [38].) (a) The ground state of $\mathrm{Pbnm} \mathrm{LiFePO}$. The AFM ordering is indicated with the black arrows. (b) Polyhedral representation of $\mathrm{LiFePO}_{4}$.

Partly motivated by a possible pressure-induced HS $\rightarrow$ LS transition in $\mathrm{LiFePO}_{4}$, we have expanded the investigated highpressure structural and vibrational behaviors of triphylite up to $80 \mathrm{GPa}$ at RT by means of synchrotron-based x-ray diffraction (XRD), infrared (IR), and Raman spectroscopies. Additionally, we have performed ab initio calculations to investigate the structural stability of $\mathrm{LiFePO}_{4}$-triphylite under pressure and the potential spin crossover in the energetically most favorable phase. By comparing the experimentally determined volume as a function of pressure to the theoretical trends throughout the pressure range considered $(0-90 \mathrm{GPa})$, we provide evidence for a pressure-induced HS-to-LS transition in the $\mathrm{Pbnm}-\mathrm{LiFePO}_{4}$ phase. After presenting our experimental (Sec. II A) and computational methods (Sec. IIB), we give comprehensive results concerning the experimental determination of vibrational and structural parameters (Secs. III A and IIIB) and computational structural and enthalpy trends (Sec. IIIC) as a function of pressure. We then discuss the spin crossover in $\mathrm{LiFePO}_{4}$ (Sec. IV) and finally present our conclusions (Sec. V). Characterization of samples, additional figures, and tables with more experimental measurements and computational results can be found in the Supplemental Material [46].

\section{METHODS}

\section{A. Experimental details}

We have investigated two synthetic $\mathrm{LiFePO}_{4}$ samples: The first one was commercial powder (Sigma-Aldrich 97\% purity, sample A), and the second sample was micrometersized single crystals (sample B) synthesized by combined high-pressure and high-temperature treatments of the aforementioned $\mathrm{LiFePO}_{4}$ powder in the multi-anvil press installed at GeoForschungsZentrum (GFZ) [47] as follows (MA502): Sample A was enclosed in a Pt capsule, whereas the 18/11 assembly was used. Pressure was raised to $1.4 \mathrm{GPa}$, then temperature was raised to $1373 \mathrm{~K}$ (melting), and the sample remained there for $30 \mathrm{~min}$. Within $2 \mathrm{~h}$, the temperature was slowly decreased down to $973 \mathrm{~K}$. The multi-anvil press was rotated for $2 \mathrm{~h}$ at this temperature. Throughout the whole experiment, the pressure was kept at $1.4 \mathrm{GPa}$.

The characterization of sample A by means of XRD and Raman spectroscopy at ambient conditions revealed the presence of two additional impurity phases at an $\sim 30 \%$ concentration (as estimated by the relative Bragg-peak intensities), i.e., the rhombohedral and monoclinic modifications of $\mathrm{Li}_{3} \mathrm{Fe}_{2}\left(\mathrm{PO}_{4}\right)_{3}$, both of them being $\mathrm{Fe}^{3+}$ bearing and common during the preparation of $\mathrm{LiFePO}_{4}$ [48]. In addition, Mössbauer spectroscopy evidenced a $30 \%$ content of $\mathrm{Fe}^{3+}$ for sample $\mathrm{A}$, in excellent agreement with our XRD measurements. On the other hand, the characterization of sample B was performed with XRD, electron microprobe analysis (EMPA), energy loss spectroscopy (EELS), and Raman and Mössbauer spectroscopies. For the EMPA measurements, several crystals of the recovered sample MA502 were selected, mounted in epoxy, polished, and coated with carbon for EMPA using a JEOL Hyperprobe JXA-8500F EMP with a field-emission cathode. As standard materials for $\mathrm{Mg}$ and $\mathrm{Si}$, we used a natural olivine, and for $\mathrm{Fe}$ and $\mathrm{P}$, we used hematite and Durango apatite, respectively. The analytical conditions included an acceleration voltage of $15 \mathrm{kV}$, a beam current of $20 \mathrm{nA}$, and a beam diameter of $1 \mu \mathrm{m}$. The $\mathrm{Li}_{2} \mathrm{O}$ content was calculated as the difference to $100 \%$. In order to check for any traces of $\mathrm{Fe}^{3+}$ in the synthesized single crystals, Mössbauer experiments were conducted on almost 100 crystals piled together on plastic with clear nail varnish and covering 400 microns in diameter. Data collection took 13 days. Finally, the presence of ferric iron in the synthetic single crystals was additionally checked with EELS. We cut a thin foil from a 
polished single crystal from the aforementioned microprobe mount using a FEI focused ion-beam 200 device. EELS were collected with a FEI Tecnai G2 F20 X-Twin TEM operating at $200 \mathrm{kV}$ with a field-emission gun electron source and a Gatan Trideim energy filter with an energy resolution of $1 \mathrm{eV}$. Details of the measurements and interpretation can be found in Ref. [49]. The combined EMPA, EELS, and Mössbauer measurements yield the following chemical composition for sample $\mathrm{B}$ : $\left(\mathrm{Li}_{0.94}, \mathrm{Fe}_{0.06}^{3+}\right)\left(\mathrm{Fe}_{0.79}^{2+}, \mathrm{Mg}_{0.18}\right) \mathrm{PO}_{4}$. More information on the characterization of the starting $\mathrm{LiFePO}_{4}$ samples A and B is provided in the Supplemental Material [46].

Gasketed diamond-anvil cells (DACs) equipped with lowfluorescence type-II diamonds of 250- and $200-\mu \mathrm{m}$ culet diameters were used for pressure generation. The rhenium gaskets were preindented to $\sim 25-\mu \mathrm{m}$ thicknesses with holes of $100-\mu \mathrm{m}$ diameters acting as sample chambers in separate runs. Argon served as the pressure-transmitting medium (PTM) in all spectroscopic experiments, whereas neon was used for the XRD measurements. The ruby-fluorescence method was employed for pressure calibration [50].

The high-pressure mid-IR (MIR) measurements at RT were conducted on $\mathrm{LiFePO}_{4}$ powder pressed into a thin film (sample A) with the Vertex 80v Fourier-transform IR spectrometer coupled to a Hyperion 2000 microscope at GFZ within the $500-1800-\mathrm{cm}^{-1}$ spectral range. We used a $\mathrm{KBr}$ beam splitter and a $\mathrm{Hg}-\mathrm{Cd}-\mathrm{Te}$ detector. The spectra were averaged over 512 scans with a spectral resolution of $2 \mathrm{~cm}^{-1}$. For details of the thin-film preparation for IR experiments, see, e.g., Ref. [51].

Our high-pressure RT Raman measurements were conducted with a Horiba Jobin Yvon LabRam HR800 VIS spectrometer at GFZ, equipped with a blue $(\lambda=473-\mathrm{nm})$ solid-state laser operating at a power of $2 \mathrm{~mW}$ outside the DAC. The same $\mathrm{LiFePO}_{4}$ thin film measured with MIR (sample A) as well as single crystals of sample B with typical dimensions of $40 \times 40 \mu \mathrm{m}^{2}$ were measured within the $100-1200-\mathrm{cm}^{-1}$ frequency region.

The angle-resolved high-pressure XRD measurements were performed at the Extreme Conditions Beamline P02.2 of PETRA III (Hamburg, Germany) [52] with an incident x-ray wavelength of $\lambda=0.289 \AA$ and a beam size of $2 \times 2 \mu \mathrm{m}^{2}$. Two-dimensional XRD patterns were collected with a fast flat panel detector XRD1621 from PerkinElmer (2048 pixels $\times$ 2048 pixels, $200 \times 200$-pixel size) and processed with the FIT2D software [53]. Refinements were performed using the GSAS + EXPGUI software packages [54]. Due to the presence of impurities in sample A as we discussed earlier, we investigated only sample B with XRD by crushing the small single crystals into powder between the diamonds of the DAC.

\section{B. Computational details}

The computational results presented in our paper were obtained by applying DFT $[39,40]$ within the projectoraugmented plane-wave (PAW) $[55,56]$ method as implemented in the VASP (version 5.4.4) software [56-58] installed in the supercomputer JURECA [59]. We employed primarily the general-gradient approximation (GGA) in the Perdew-BurkeErnzerhof (PBE) [60] prescription for the exchange-correlation potential, and for comparison we also tested the local-density approximation (LDA) [61]. The default PAW potentials with

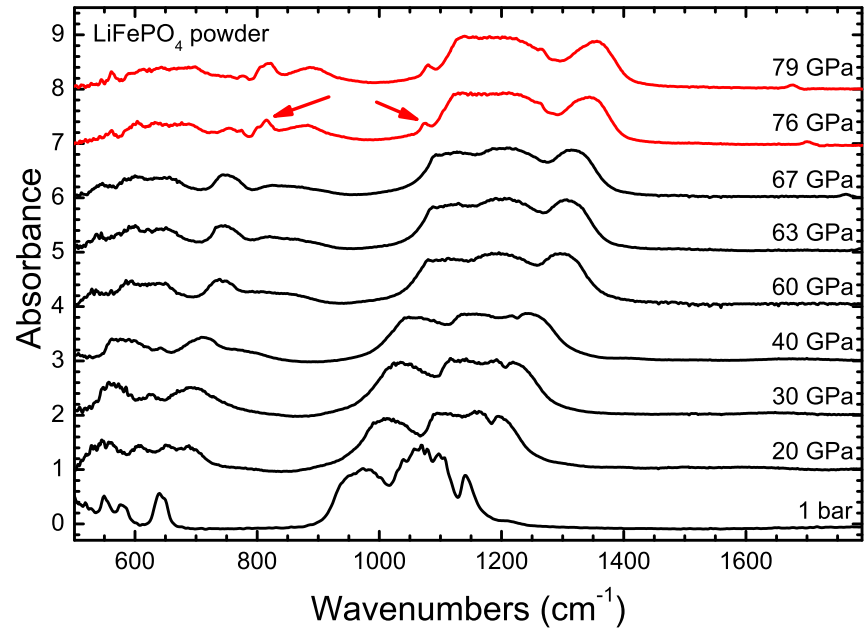

FIG. 2. MIR spectra of $\mathrm{LiFePO}_{4}$ sample A at various pressures. The black and red spectra correspond to the Pbnm and the HP-Pbnm phases, respectively. The red arrows indicate the new MIR bands (see the text).

the valence configurations of $1 s^{2} 2 s^{1}$ for lithium, $3 p^{6} 3 d^{7} 4 s^{1}$ for iron, $3 s^{2} 3 p^{3}$ for phosphorous, and $2 s^{2} 2 p^{4}$ for oxygen were used throughout our calculations. We adopted a plane-wave kinetic-energy cutoff of $500 \mathrm{eV}$ and a uniform $\Gamma$-centered grid with $(2 \pi \times 0.032)-\AA^{-1}$ spacing for the reciprocal space sampling of our 28 -atom cells for pressures between -5 and $100 \mathrm{GPa}$. The Hubbard- $U$ correction $[62,63]$ was added to the localized Fe $3 d$ electrons (LDA $+U$ and $\mathrm{PBE}+U$ ), and we assessed its value in the range of $0-5.3 \mathrm{eV}$ but found $U=2.5 \mathrm{eV}$ to better agree with the experimentally determined lattice parameters and the pressure at which evidence of a spin transition is observed in $\mathrm{LiFePO}_{4}$ (see Figs. 5 and 6 in the Supplemental Material [46]). Convergence of our calculations at each pressure was assumed when the forces on each atom were smaller than $1 \mathrm{meV} / \AA$ and the total energy changes less than $10^{-8} \mathrm{eV}$. Triphylite structures were set up at each target pressure within the Pbnm and $\mathrm{Cmcm}$ space groups and subsequently fully relaxed (internal coordinates and lattice parameters) using a conjugate gradient minimization approach. At zero pressure, the ordering of the spin of $\mathrm{Fe}^{2+}$, i.e., $\mathrm{AFM}$ and ferromagnetic (FM) configurations, was also probed.

\section{RESULTS}

\section{A. Vibrational spectroscopy under pressure}

According to group theory [64-66], the expected IR- and Raman-active modes in $\mathrm{LiFePO}_{4}$ are as follows:

$$
\begin{aligned}
\Gamma= & 11 A_{g}+11 B_{1 g}+7 B_{2 g}+7 B_{3 g}+14 B_{1 u} \\
& +14 B_{2 u}+10 B_{3 u} .
\end{aligned}
$$

Thus, $\mathrm{LiFePO}_{4}$ has 36 Raman-active modes $\left(11 A_{g}+11 B_{1 g}+\right.$ $\left.7 B_{2 g}+7 B_{3 g}\right)$ and 38 IR-active modes $\left(14 B_{1 u}+14 B_{2 u}+\right.$ $\left.10 B_{3 u}\right)$. In Fig. 2, we present selected high-pressure MIR spectra collected for sample A. The ambient-pressure MIR spectrum of $\mathrm{LiFePO}_{4}$ is consistent with the reported literature [67,68]. The intense broad MIR bands located between 1000 and $1200 \mathrm{~cm}^{-1}$ correspond to internal antisymmetric 
TABLE I. Volume $V$, bulk modulus $B$, its pressure derivative $B^{\prime}$ for $P b n m \mathrm{LiFePO}_{4}$, and its $\mathrm{HP}-\mathrm{Pbnm}$ phase as obtained by a BirchMurnaghan EoS [70] fitted to our measured and computed $P-V$ data ${ }^{\diamond}$ "Fixed" values were not allowed to vary during the fitting. Other results are from ${ }^{\star}$ : Ref. [71]; ${ }^{\dagger}$ : Ref. [44]; ${ }^{\ddagger}$ : Ref. [43].

\begin{tabular}{|c|c|c|c|c|c|}
\hline $\mathrm{LiFePO}_{4}$ phase & Method & $P(\mathrm{GPa})$ & $V\left(\AA^{3}\right)$ & $B(\mathrm{GPa})$ & $B^{\prime}(\mathrm{GPa})$ \\
\hline \multirow[t]{7}{*}{ Pbnm } & $\mathrm{EXP}^{\diamond}$ & 0.0 & 290.80 (fixed) & $99.0(1)$ & 4.0 (fixed) \\
\hline & $\mathrm{GGA}+U^{\diamond}$ & 0.0 & 299.00 & 90.9 & 4.3 \\
\hline & $\mathrm{GGA}+U^{\star}$ & 0.0 & 299.54 & 94.7 & \\
\hline & $\mathrm{EXP}^{\dagger}$ & 0.0 & 292.38 & 91.5 & 4.0 (fixed) \\
\hline & $\mathrm{GGA}+U^{\dagger}$ & 0.0 & 292.38 & 92.9 & \\
\hline & $\mathrm{LDA}+U^{\dagger}$ & 0.0 & 300.62 & 114.1 & \\
\hline & $\mathrm{EXP}^{\ddagger}$ & 0.0 & 291.60 & $106.0(8)$ & \\
\hline HP-Pbnm & $\mathrm{EXP}^{\diamond}$ & 73.0 & 203.00 (fixed) & $545.0(2)$ & 4.0 (fixed) \\
\hline HP-Pbnm (LS) & $\mathrm{GGA}+U^{\diamond}$ & 73.0 & 196.47 & 381.0 & 4.0 \\
\hline
\end{tabular}

P-O stretching vibrations $v_{3}$, the broadband centered at $\sim 960 \mathrm{~cm}^{-1}$ belongs to the symmetric P-O stretching motion $v_{1}$, and the IR region between 400 and $800 \mathrm{~cm}^{-1}$ is mainly attributed to the internal bending motions of the $\mathrm{PO}_{4}$ tetrahedra $v_{2}$ and $v_{4}[64,67,68]$. The complete assignment can be found in Table I in the Supplemental Material [46].

Increasing pressure leads to the frequency upshift and a broadening of the recorded MIR bands, owing partly to the nonhydrostatic conditions imposed by the Ar PTM upon increasing pressure [69] as well as the polycrystalline form of our sample. Nevertheless, close to $70 \mathrm{GPa}$ we can detect the appearance of new IR bands between 700 and $800 \mathrm{~cm}^{-1}$ and at $1000 \mathrm{~cm}^{-1}$, indicating a pressure-induced transition of the Pbnm-LiFePO ${ }_{4}$ structure. We will identify this high-pressure modification as HP-Pbnm from now on. Interestingly, we can observe that the broadband at $750 \mathrm{~cm}^{-1}$ is apparently vanishing in the new phase with no other significant changes taking place throughout the transition.

Concurrently with our MIR experiment, we performed Raman spectroscopic investigations on the same $\mathrm{LiFePO}_{4}$ thin film (sample A). Selected Raman spectra at various pressures are shown in Fig. 3(a). We note that the strongest Raman peak located at $950 \mathrm{~cm}^{-1}$ corresponds to the symmetric $\mathrm{P}-\mathrm{O}$ $v_{1}$ stretching vibration $[64,66]$. Increasing pressure leads to the appearance of a small shoulder in the vicinity of the aforementioned $v_{1}$ mode at $67 \mathrm{GPa}$, indicating the phase transition of $\mathrm{LiFePO}_{4}$ towards $\mathrm{HP}-\mathrm{Pbnm}$ at this pressure and in excellent agreement with our MIR results. Further compression results in the intensity enhancement of this shoulder at the expense of the $950-\mathrm{cm}^{-1}$ peak. Moreover, we can observe the

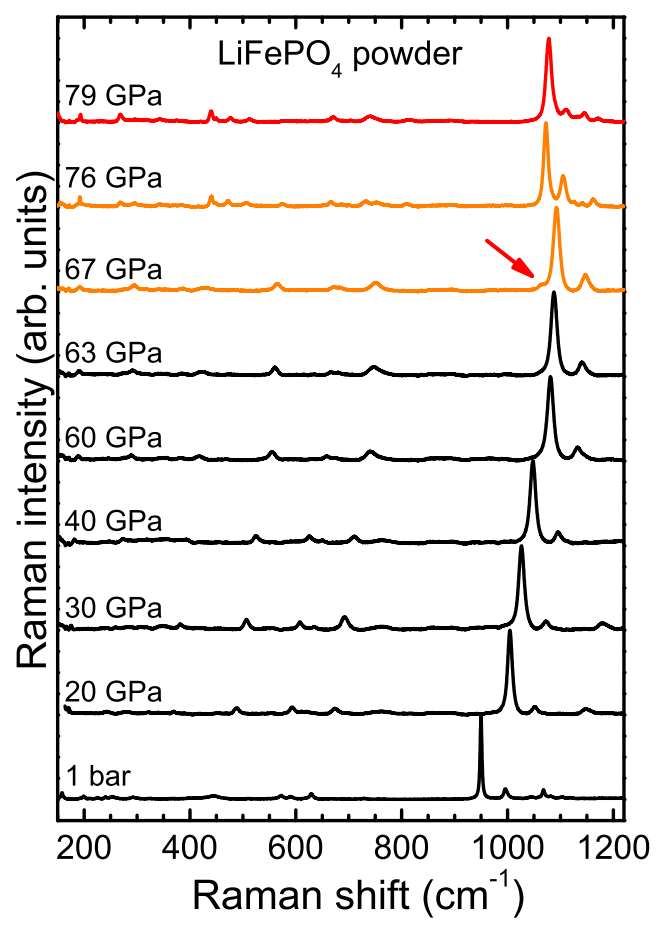

(a)

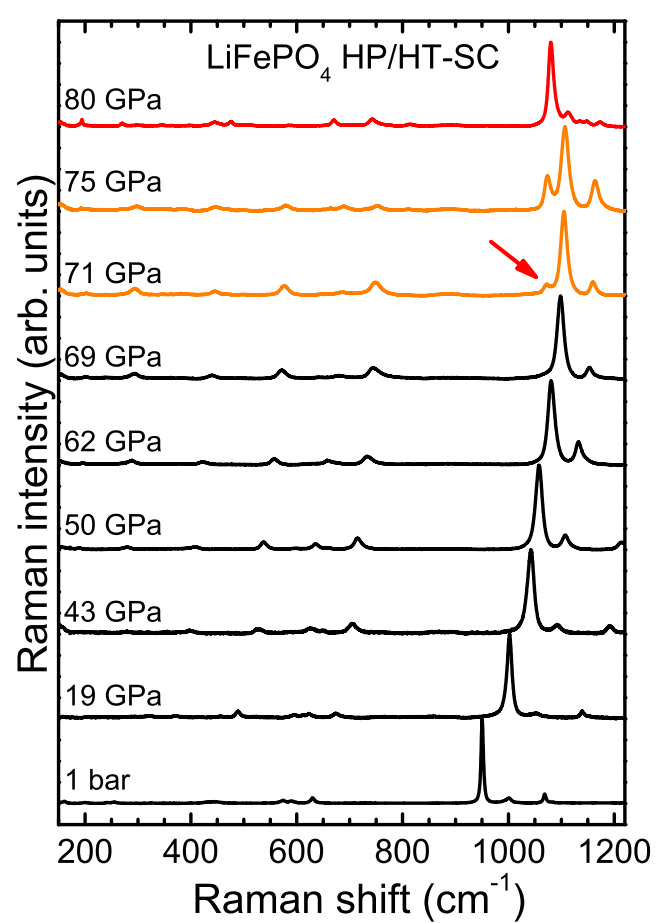

(b)

FIG. 3. Raman spectra of $\mathrm{LiFePO}_{4}$ (a) sample A and (b) sample B at selected pressures. The black, red, and orange spectra correspond to the Pbnm, the HP-Pbnm phases, and the coexistence range, respectively. The red arrows indicate the new Raman features in both cases (see the text). 
appearance of several low-intensity Raman features (Fig. 4 in the Supplemental Material [46]).

In order to test whether this pressure-induced transition is possibly a by-product of the polycrystalline nature of our sample or its impurities (see the Supplemental Material [46]), we have additionally conducted high-pressure Raman investigations on the (HP/HT)-synthesized $\mathrm{LiFePO}_{4}$ single crystals. The relevant Raman spectra are plotted in Fig. 3(b). As we can observe clearly, the situation is identical with that of sample A, i.e., a new Raman peak in the vicinity of the strong 950- $\mathrm{cm}^{-1}$ mode emerges at $71 \mathrm{GPa}$. We note that the Raman spectra at $\sim 80 \mathrm{GPa}$ for both samples are practically identical, indicating that the pressure-induced Pbnm $\rightarrow$ HP-Pbnm structural transition of $\mathrm{LiFePO}_{4}$ is inherent to the material and independent of the starting condition of the sample. At this stage, we cannot assign the high-pressure modification to the predicted nonmagnetic $\mathrm{Cmcm}$ phase (calculated to be adopted close to $52 \mathrm{GPa}$ ) [41] as its Raman spectrum has not been reported.

\section{B. Structure under pressure}

Following our vibrational spectroscopic results, we have additionally conducted high-pressure XRD experiments in order to identify the aforementioned phase transition close to $70 \mathrm{GPa}$. We have only probed the structural behavior of sample B since the Bragg peaks of the impurities in sample A (see Sec. II A as well as the Supplemental Material [46]) might hinder the clear detection of a structural change. Overall, the XRD patterns collected up to $79 \mathrm{GPa}$ reveal indeed a transition initiating at $69 \mathrm{GPa}$ as exposed by the appearance of a shoulder in the Bragg peak centered at $2 \theta \simeq 7^{\circ}$ [Fig. 4(a)]. Further compression leads to the enhancement of this shoulder at the expense of the $7^{\circ}$ Bragg peak attributed to the starting Pbnm phase.

Indexing of the XRD pattern at $78.5 \mathrm{GPa}$ is possible again with a Pbnm structure [Fig. 4(b)]. Hence, $\mathrm{LiFePO}_{4}$ undergoes an isostructural transition close to $70 \mathrm{GPa}$; the transition pressure lies in excellent agreement with our vibrational studies discussed above. According to our extracted lattice parameters, in Fig. 5(a), upon passing from the Pbnm to the HP-Pbnm phase, the orthorhombic $\mathbf{b}$ axis decreases by $\sim 3 \%$, which in turn results in an overall volume reduction of $\sim 3 \%$ (Fig. 5). Thus, the isostructural Pbnm $\rightarrow$ HP-Pbnm transition can be classified as of first order. We should also note that we tested the proposed $\mathrm{Cmcm}$ phase [41], but it could not reproduce the observed high-pressure XRD patterns.

In Fig. 5 we plot the measured pressure-volume $(P-V)$ data alongside the respective EoS fittings and our GGA+U (with $U=2.5-\mathrm{eV}$ ) results. The extracted elastic parameters are listed in Table I. As we can observe, our volume at zero pressure $V_{0}$ and bulk modulus $B_{0}$ values for the starting $P$ bnm phase of $\mathrm{LiFePO}_{4}$ are in good agreement with the reported literature $[43,44,71]$.

\section{Structural relaxations}

In order to complement and verify our experimental results, we also performed DFT calculations. For this purpose, first we stabilized the AFM and FM configurations of Pbnm
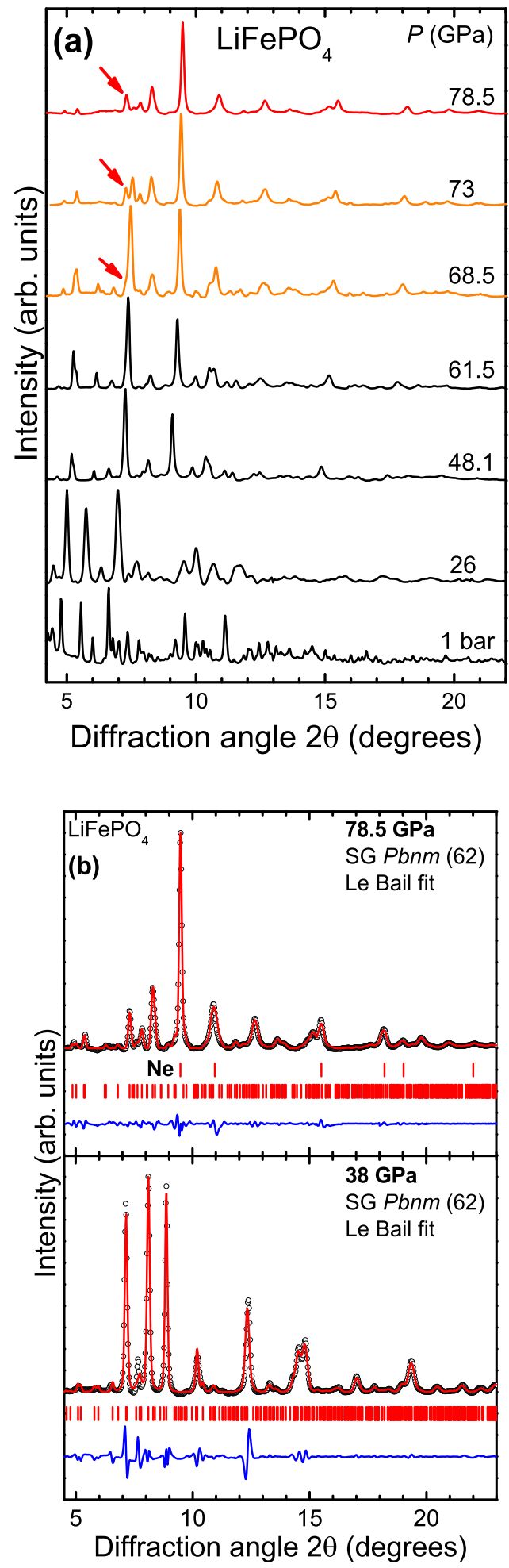

FIG. 4. (a) Selected XRD patterns of $\mathrm{LiFePO}_{4}$ (sample B) at various pressures $(T=300 \mathrm{~K}, \lambda=0.29 \AA)$. The $P b n m$ phase is shown in black, the HP-Pbnm phase is shown in red, and their coexistence range is depicted in orange. The red arrows indicate the new XRD features (see the text). (b) Examples of Le Bail refinements at $38 \mathrm{GPa}$ (bottom) and $78.5 \mathrm{GPa}$ (top). The black circles and the red solid lines correspond to the measured and the fitted patterns, whereas their difference is depicted as a blue line. The vertical ticks mark the Bragg-peak positions for the Pbnm phase and the neon PTM (only the top). 


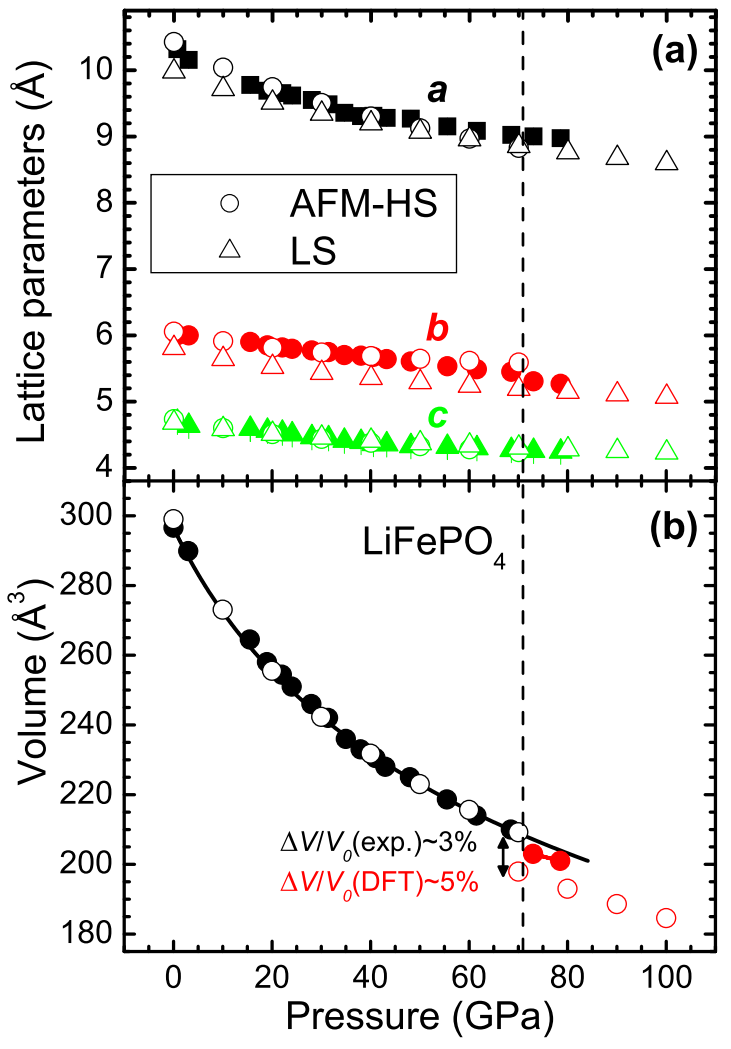

FIG. 5. (a) Lattice parameters (EXP: the solid symbols; DFT + $U$ : the open symbols) and (b) unit-cell volume as a function of pressure for $P$ bnm $\mathrm{LiFePO}_{4}$ ( $\lesssim 73 \mathrm{GPa}$; EXP: the solid black circles; DFT + $U$ : the open black circles) and its HP-Pbnm phase ( $\gtrsim 73 \mathrm{GPa}$; EXP: the solid red circles; DFT $+U$ : the open red circles). The solid lines running through the symbols are the fitted Birch-Murnaghan equation of state (EoS) forms. The vertical dashed line marks the onset of the isostructural transition. Error bars lie within the symbols.

and $\mathrm{Cmcm} \mathrm{LiFePO}_{4}$ at ambient pressure and determined the

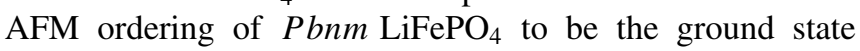
at $\sim 7.5$ and $\sim 12.5 \mathrm{meV}$ per formula unit (f.u.) lower than the FM Pbnm $\mathrm{LiFePO}_{4}$ and the AFM Cmcm $\mathrm{LiFePO}_{4}$ for $U=4.3 \mathrm{eV}$, respectively. We began with $U=4.3 \mathrm{eV}$ as this value was used in the most recent work on the stability of triphylite [44], and although $U$ seems to be not considered by Lin and Zeng [41] in their Pbnm $\rightarrow \mathrm{Cmcm}$ phase-transition study, the authors also report AFM ordering as the ground states for their Pbnm-and Cmcm-triphylite phases. We note that AFM Pbnm $\mathrm{LiFePO}_{4}$ holds as the ground state independent of $U(=0,2.5,3.3,4.3,5.3 \mathrm{eV})$. The energy differences we found are consistent with first-principles calculations also performed in $\mathrm{LiFePO}_{4}$ as part of a study of Li compounds [72] at ambient conditions. Thus, in the rest of the paper we only examine AFM phases with $U=2.5 \mathrm{eV}$ as it was the value to produce the most compatible structural parameters and description of the high-pressure spin state of $\mathrm{LiFePO}_{4}$ (Fig. 5).

Our static DFT results for the total internal energy $(E)$ per f.u. as a function of volume per f.u. of the AFM Pbnm- and $\mathrm{Cmcm}$-triphylite phases are shown in Fig. 6. As one can see, the equations of state of these two phases are nearly identical for high pressures. To further assess the stability of the orthorhom-

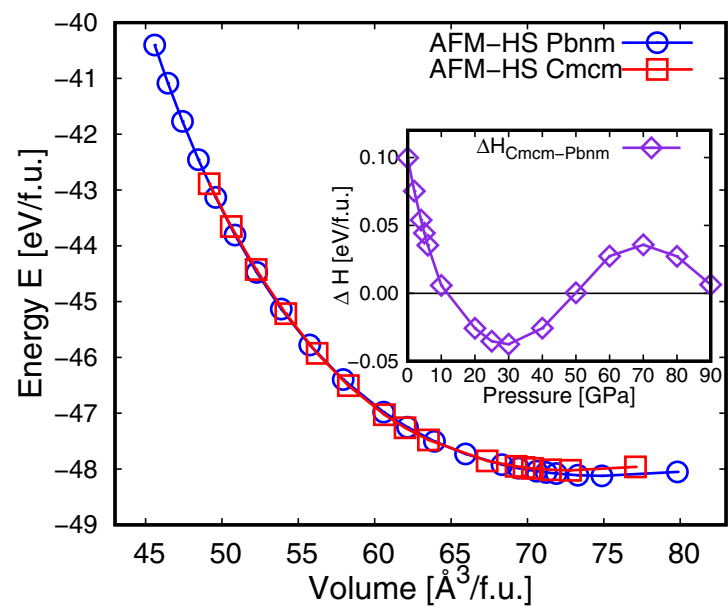

FIG. 6. Calculated total internal energy per f.u. as a function of volume per f.u. for the two AFM structures of $\mathrm{LiFePO}_{4}$ considered, $P b n m$ and $\mathrm{Cmcm}$. The inset: Stability of these phases as shown by their relative enthalpies as a function of pressure.

bic $P b n m$ - $\mathrm{LiFePO}_{4}$ phase with respect to $C m c m \mathrm{LiFePO}_{4}$, we calculated their enthalpy $(H)$ as a function of pressure $(P)$, volume $(V)$, and internal energy $(E)$ at each $P$,

$$
H(P)=E[V(P)]+P V(P) .
$$

We found, that as pressure increases, as shown in the inset of Fig. 6, the difference in enthalpy per f.u. $(\Delta H)$ between the $P b n m$ and the $C m c m$ phases fluctuates for $P>10 \mathrm{GPa}$, i.e., $-0.04 \mathrm{eV}<\Delta H<0.04 \mathrm{eV}$, making these two competing phases almost degenerate within the accuracy of our static first-principles method. However, upon comparison with the structural XRD refinements [Fig. 4(a)], the Pbnm structure is favored in this pressure range. Therefore, we conclude that the proposed pressure-induced $\mathrm{Pbnm} \rightarrow \mathrm{Cmcm}$ phase transition in $\mathrm{LiFePO}_{4}$ [41] is not likely at RT. Further examination contrasting our experimental and calculated lattice parameters of $\mathrm{LiFePO}_{4}$-triphylite shows, in general, an excellent agreement for the starting AFM-HS Pbnm phase $(\lesssim 75 \mathrm{GPa})$, Fig. 5(a) (see also Fig. 5 and Tables III-V in the Supplemental Material [46]). Upon reaching the transition pressure point, we notice that the magnitude of the lattice parameter along the $\mathbf{b}$ axis undergoes the largest decrease thus contributing greatly to the volume drop (Fig. 5) observed experimentally $(\sim 3 \%)$ and predicted by GGA $+U(\sim 5 \%)$. We should note here that the modest agreement between the experimental and the calculated volume reductions across the HS-to-LS transition in $\mathrm{LiFePO}_{4}$ can be partially attributed to the sensitive nature of the DFT $+U$ method. In particular, it has been established that the choice of $U$ can significantly affect both the spin transition pressure as well as the volume difference between the two phases. For example, in the case of $\mathrm{FeCO}_{3}$, within the GGA $+U$ approximation, $U=0 \mathrm{eV}$ results in a transition pressure of $15 \mathrm{GPa}$ and a volume reduction of $12.5 \%$, whereas $U=4 \mathrm{eV}$ shifts the transition pressure to $28 \mathrm{GPa}$ and the volume difference to $\sim 10 \%$ [73]; furthermore, using a self-consistent $U$ parameter $(\mathrm{GGA}+U)$ gives a transition pressure range of $47-50 \mathrm{GPa}$ and a volume drop of $\sim 10 \%$ at the transition point [34]. These results, still contrast, to 


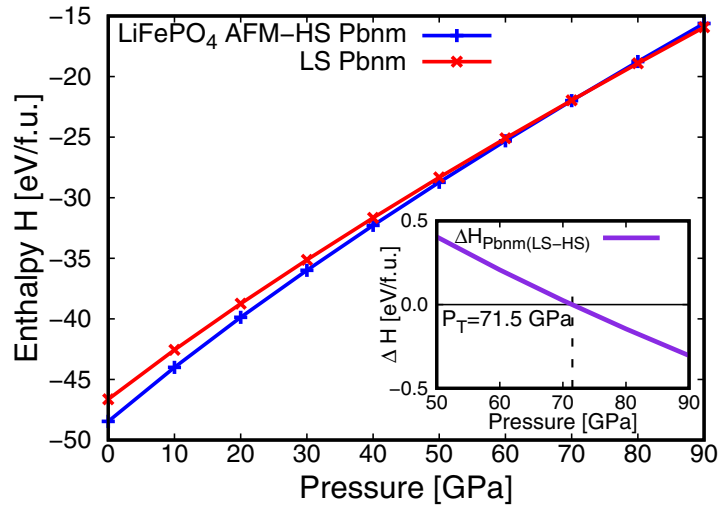

FIG. 7. Enthalpy of $\mathrm{Pbnm}_{\mathrm{LiFePO}}$ in HS and LS states as a function of pressure. The inset shows the spin transition pressure $\left(P_{T}\right)$ as predicted from GGA $+U$ with $U=2.5 \mathrm{eV}$.

a varying degree, the experiments on $\mathrm{FeCO}_{3}$, which exhibit transition pressures within the 43-52-GPa pressure range, and the corresponding volume reductions varying between $6.5 \%$ and $10 \%$ [28-30]. In our case, if we choose GGA $+U$ with $U=4.3 \mathrm{eV}$ (see Figs. 5 and 6 in the Supplemental Material [46]), we obtain a volume reduction of $4 \%$, which is in much better agreement with the collapsed volume observed in our experiments of $\sim 3 \%$, but this outcome is at the cost of an increase in the HS $\rightarrow$ LS transition pressure with respect to the one experimentally determined. Additionally, we also have to consider that our DFT computations were performed at $0 \mathrm{~K}$, whereas the experiment was performed at room temperature. Given also the fact that the chemical composition of our experimental single-crystalline sample is not stoichiometric $\mathrm{LiFePO}_{4}$ as in our calculations but rather a slightly more complicated chemical formula, i.e., $\left(\mathrm{Li}_{0.94}, \mathrm{Fe}_{0.06}^{3+}\right)\left(\mathrm{Fe}_{0.79}^{2+}, \mathrm{Mg}_{0.18}\right) \mathrm{PO}_{4}$, we can expect differences in the exact volume reduction at the HS-to-LS transition point, similar to the $\left(\mathrm{Fe}_{x}, \mathrm{Mg}_{1-x}\right) \mathrm{CO}_{3}$ solid solution series [30].

\section{SPIN TRANSITION}

As we have already mentioned in Sec. I, high-pressure studies of Fe-bearing minerals have established a HS-LS transition upon sufficient compression $[4,18,26]$. This magnetic change is usually accompanied by a first-order isostructural transition, i.e., the crystalline symmetry is retained during the HS-LS transition, whereas the first-order character is attributed to the reduction of the $\mathrm{Fe}^{2+}$ ionic radius upon the spin change [74]. Using DFT we studied the spin state behavior of $P b n m \mathrm{LiFePO}_{4}$ as a function of pressure within GGA $+U$ with $U=2.5 \mathrm{eV}$. We stabilized $P$ bnm $\mathrm{LiFePO}_{4}$ in its LS state and computed its enthalpy [Eq. (2)] to compare it to that of Pbnm $\mathrm{LiFePO}_{4}$ in the AFM-HS configuration for pressures between 0 and $90 \mathrm{GPa}$. Figure 7 shows the switch in spin state from HS to LS at about $72 \mathrm{GPa}$, in excellent agreement with the observed transition pressure in our experiments. To further corroborate the presence of this spin crossover, we also investigated the relative enthalpies of the HS and LS states using other $U$ values within the GGA $+U$ and LDA $+U$ schemes. Our results, detailed in our Supplemental Material [46], indicate that the spin transition occurs for all values of

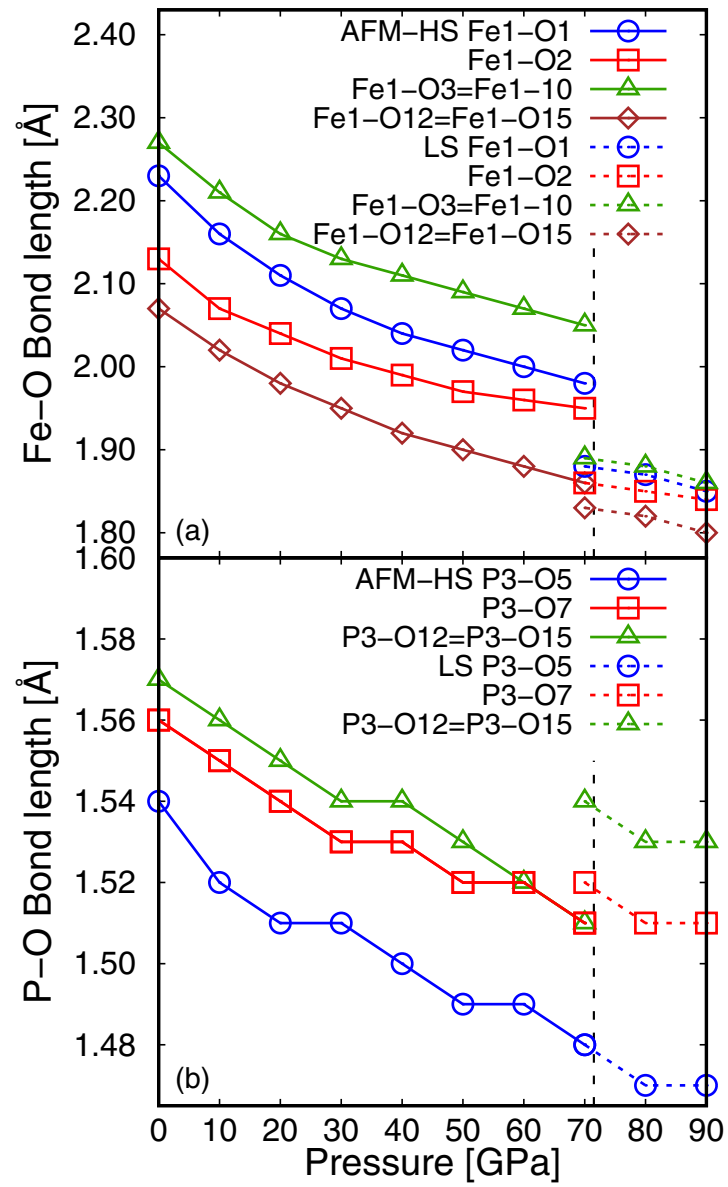

FIG. 8. Computed (a) Fe1-O and (b) P3-O bond length distances as a function of pressure for $\mathrm{Pbnm} \mathrm{LiFePO}_{4}$ in $\mathrm{HS}$ and LS states within GGA $+U$ with $U=2.5 \mathrm{eV}$. The vertical dashed lines mark the onset of the isostructural transition. Bond lengths for $\mathrm{Fe} 2-\mathrm{O}, \mathrm{Fe} 3-$ $\mathrm{O}, \mathrm{Fe} 4-\mathrm{O}, \mathrm{P} 1-\mathrm{O}, \mathrm{P} 2-\mathrm{O}$, and $\mathrm{P} 3-\mathrm{O}$ are analogous (see Fig. 1).

$U$ tested (Fig. 5 in the Supplemental Material [46]) and that the spin transition pressure predicted by GGA $+U$ increases with $U$. LDA $+U$ also showed this spin crossover, however, the structural parameters and volume of triphylite computed within this scheme were grossly underestimated (see Fig. 6 in the Supplemental Material [46]).

In order to gain a microscopic structural understanding of the HS-to-LS transition in $\mathrm{LiFePO}_{4}$, we plot selected ab initio $\mathrm{Fe}-\mathrm{O}$ and P-O bond lengths as a function of pressure (Fig. 8). As we can clearly observe in Fig. 8(a), all the Fe-O bond distances shrink abruptly at the transition point, following the decrease in the $\mathrm{Fe}^{2+}$ ionic radius upon the spin crossover. This effect has been previously observed in other Fe-bearing compounds $[27,28,37]$. At the same time, we note that three of the P-O bonds increase across the transition pressure, Fig. 8(b). This $\mathrm{P}-\mathrm{O}$ bond enlargement lies in excellent agreement with our Raman investigations where a frequency downshift of the $v_{1}$ mode was observed at the transition point (Fig. 3). Since the $v_{1}$ mode corresponds to the symmetric $\mathrm{P}-\mathrm{O}$ stretching motion [64,67], its frequency downshift implies an increase in the associated P-O bonds at the spin crossover. Our DFT $+U$ calculations have indeed captured this effect. 
Considering the very good consistency between our experimental and computational results, we can establish at this point that $\mathrm{LiFePO}_{4}$ undergoes a spin-driven isostructural transition at $\sim 70 \mathrm{GPa}$ with its magnetic configuration changing from AFM to nonmagnetic owing to the pressureinduced HS-LS transition. Compared to relevant Fe-bearing minerals [4-34,36], $\mathrm{LiFePO}_{4}$ exhibits the highest onset pressure for a spin transition to date. A plausible explanation might be the incompressibility of the $\mathrm{FeO}_{6}$ octahedra in the olivine structure compared to, e.g., the ferropericlase and carbonate structures. In Fig. 8 we can see that as pressure increases, the $\mathrm{Fe}-\mathrm{O}$ bond-length distance first decreases rather rapidly but at about $40 \mathrm{GPa}$, the contraction rate decreases.

Finally, we would like to add some thoughts on the possible effect of the HS-to-LS transition on the $\mathrm{LiFePO}_{4}$ performance as a cathode material, i.e., on the $\mathrm{Li}^{+}$diffusion properties. The effect of pressure on the $\mathrm{Li}^{+}$diffusion rate has been modeled up to $28 \mathrm{GPa}$ [44]. These calculations showed that the $\mathrm{Li}^{+}$migration barrier energy doubles along each possible diffusion direction, i.e., the volume reduction hinders the $\mathrm{Li}^{+}$ diffusion and, consequently, diminishes the $\mathrm{LiFePO}_{4}$ battery performance. Furthermore, we can reasonably assume that a potential pressure-induced $\mathrm{Fe}^{2+}$ spin transition might reduce the $\mathrm{LiFePO}_{4}$ cathode efficiency even further, following the smaller unit-cell volume of the LS configuration compared to the HS state as a result of the $\mathrm{Fe}^{2+}$ ionic radius reduction. Another preventive factor might be the empty $\mathrm{Fe}^{2+} e_{g}$ states of the LS state; such a conclusion was reached for perovskite oxides [75]. On the other hand, the symmetrization of the Fe-O bond lengths upon the HS-to-LS transition (Fig. 8) may enhance the $\mathrm{Li}^{+}$mobility in $\mathrm{LS}-\mathrm{LiFePO}_{4}$, partially counteracting the aforementioned hindering factors of battery efficiency and performance in this particular material [76].

\section{CONCLUSIONS}

We have investigated the olivine-type $\mathrm{LiFePO}_{4}$ structural and magnetic properties under pressure with a combination of first-principles and experimental probes. Our investigations indicate that the starting $\mathrm{Pbnm}$ phase of $\mathrm{LiFePO}_{4}$ persists up to $\sim 70 \mathrm{GPa}$. Further compression leads to a first-order isostructural transition in the pressure range of $\sim 70-75 \mathrm{GPa}$, inconsistent with previous claims regarding the adoption of a $\mathrm{Cmcm}$ structure at $\sim 4 \mathrm{GPa}$ [41]. Considering our DFT results placing a high-spin-to-low-spin transition of $\mathrm{Fe}^{2+}$ close to $72 \mathrm{GPa}$, we attribute the experimentally observed isostructural transition to a change in the spin state of $\mathrm{Fe}^{2+}$ in $\mathrm{LiFePO}_{4}$, i.e., a transition from the starting AFM-Pbnm phase towards a nonmagnetic state. To date, our results imply that $\mathrm{LiFePO}_{4}$ exhibits the highest onset pressure for a spin state transition among Fe-bearing minerals, resulting most likely from the larger incompressibility of its $\mathrm{FeO}_{6}$ octahedra.

\section{ACKNOWLEDGMENTS}

We thank Dr. R. Wirth for his assistance with the EELS measurements. This work was supported by the Deutsche Forschungs Gemeinschaft (DFG) Funds No. Ko1260/18 and No. Wi2000/10. Parts of this research were carried out at the light source PETRA III at DESY, a member of the Helmholtz Association (HGF). M.N.V. gratefully acknowledges the computing time granted by the John von Neumann Institute for Computing (NIC) and provided on the supercomputer JURECA at Jülich Supercomputing Centre (JSC) under Project No. ID HPO24. Some computations were also performed at the GFZ linux cluster GLIC.
[1] A. Losey, J. Rakovan, J. M. Hughes, C. A. Francis, and M. D. Dyar, Can. Mineral. 42, 1105 (2004).

[2] K. Langer, M. N. Taran, and A.-M. Fransolet, Eur. J. Mineral. 18, 337 (2006).

[3] A. E. Ringwood, Composition and Petrology of the Earth's Mantle (McGraw-Hill, New York, 1975), p. 618.

[4] J. Badro, Science 300, 789 (2003).

[5] J.-F. Lin, V. V. Struzhkin, S. D. Jacobsen, M. Y. Hu, P. Chow, J. Kung, H. Liu, H.-k. Mao, and R. J. Hemley, Nature (London) 436, 377 (2005).

[6] S. Speziale, A. Milner, V. E. Lee, S. M. Clark, M. P. Pasternak, and R. Jeanloz, Proc. Natl. Acad. Sci. USA 102, 17918 (2005).

[7] T. Tsuchiya, R. M. Wentzcovitch, C. R. S. da Silva, and S. de Gironcoli, Phys. Rev. Lett. 96, 198501 (2006).

[8] A. F. Goncharov, V. V. Struzhkin, and S. D. Jacobsen, Science 312, 1205 (2006).

[9] J.-F. Lin, G. Vanko, S. D. Jacobsen, V. Iota, V. V. Struzhkin, V. B. Prakapenka, A. Kuznetsov, and C.-S. Yoo, Science 317, 1740 (2007).

[10] J. C. Crowhurst, J. M. Brown, A. F. Goncharov, and S. D. Jacobsen, Science 319, 451 (2008).
[11] R. M. Wentzcovitch, J. F. Justo, Z. Wu, C. R. S. da Silva, D. A. Yuen, and D. Kohlstedt, Proc. Natl. Acad. Sci. USA 106, 8447 (2009).

[12] H. Marquardt, S. Speziale, H. J. Reichmann, D. J. Frost, F. R. Schilling, and E. J. Garnero, Science 324, 224 (2009).

[13] D. Antonangeli, J. Siebert, C. M. Aracne, D. L. Farber, A. Bosak, M. Hoesch, M. Krisch, F. J. Ryerson, G. Fiquet, and J. Badro, Science 331, 64 (2011).

[14] Z. Wu, J. F. Justo, and R. M. Wentzcovitch, Phys. Rev. Lett. 110, 228501 (2013).

[15] E. Holmström and L. Stixrude, Phys. Rev. Lett. 114, 117202 (2015).

[16] Z. Wu and R. M. Wentzcovitch, Proc. Natl. Acad. Sci. USA 111, 10468 (2014).

[17] H. Hsu, K. Umemoto, Z. Wu, and R. M. Wentzcovitch, Rev. Mineral. Geochem. 71, 169 (2010).

[18] J. Badro, Science 305, 383 (2004).

[19] J. Li, V. V. Struzhkin, H.-K. Mao, J. Shu, R. J. Hemley, Y. Fei, B. Mysen, P. Dera, V. Prakapenka, and G. Shen, Proc. Natl. Acad. Sci. USA 101, 14027 (2004).

[20] C. McCammon, I. Kantor, O. Narygina, J. Rouquette, U. Ponkratz, I. Sergueev, M. Mezouar, V. Prakapenka, and L. Dubrovinsky, Nat. Geosci. 1, 684 (2008). 
[21] J.-F. Lin, H. Watson, G. Vankó, E. E. Alp, V. B. Prakapenka, P. Dera, V. V. Struzhkin, A. Kubo, J. Zhao, C. McCammon, and W. J. Evans, Nat. Geosci. 1, 688 (2008).

[22] H. Hsu, K. Umemoto, P. Blaha, and R. M. Wentzcovitch, Earth Planet. Sci. Lett. 294, 19 (2010).

[23] H. Hsu, P. Blaha, M. Cococcioni, and R. M. Wentzcovitch, Phys. Rev. Lett. 106, 118501 (2011).

[24] V. Potapkin, C. McCammon, K. Glazyrin, A. Kantor, I. Kupenko, C. Prescher, R. Sinmyo, G. V. Smirnov, A. I. Chumakov, R. Rüffer, and L. Dubrovinsky, Nat. Commun. 4, 1427 (2013).

[25] H. Hsu and R. M. Wentzcovitch, Phys. Rev. B 90, 195205 (2014).

[26] A. Mattila and T. Pylkkänen, J. Phys.: Condens. Matter 19, 386206 (2007).

[27] B. Lavina, P. Dera, R. T. Downs, V. Prakapenka, M. Rivers, S. Sutton, and M. Nicol, Geophys. Res. Lett. 36, 2 (2009).

[28] B. Lavina, P. Dera, R. T. Downs, W. Yang, S. Sinogeikin, Y. Meng, G. Shen, and D. Schiferl, Phys. Rev. B 82, 064110 (2010).

[29] T. Nagai, T. Ishido, Y. Seto, D. Nishio-Hamane, N. Sata, and K. Fujino, J. Phys.: Conf. Ser. 215, 012002 (2010).

[30] G. Farfan, S. Wang, H. Ma, R. Caracas, and W. L. Mao, Am. Mineral. 97, 1421 (2012).

[31] J. F. Lin, J. Liu, C. Jacobs, and V. B. Prakapenka, Am. Mineral. 97, 583 (2012).

[32] J. Liu, J.-F. Lin, Z. Mao, and V. B. Prakapenka, Am. Mineral 99, 84 (2014).

[33] S. S. Lobanov, A. F. Goncharov, and K. D. Litasov, Am. Mineral. 100, 1059 (2015).

[34] H. Hsu and S. C. Huang, Phys. Rev. B 94, 060404 (2016).

[35] J. Müller, S. Speziale, I. Efthimiopoulos, S. A. Jahn, and M. Koch-Müller, Am. Mineral. 101, 2638 (2016).

[36] J. Müller, I. Efthimiopoulos, S. A. Jahn, and M. Koch-Müller, Eur. J. Mineral. 29, 785 (2017).

[37] J.-F. Lin, S. Speziale, Z. Mao, and H. Marquardt, Rev. Geophys. 51, 244 (2013).

[38] K. Momma and F. Izumi, J. Appl. Cryst. 41, 653 (2008).

[39] P. Hohenberg and W. Kohn, Phys. Rev. 136, B864 (1964).

[40] W. Kohn and L. J. Sham, Phys. Rev. 140, A1133 (1964).

[41] H. Lin and Z. Zeng, IEEE Trans. Magn. 47, 3817 (2011).

[42] O. García-Moreno, M. Alvarez-Vega, F. García-Alvarado, J. García-Jaca, J. M. Gallardo-Amores, M. L. Sanjuán, and U. Amador, Chem. Mater. 13, 1570 (2001).

[43] J. L. Dodd, Phase Composition and Dynamical Studies of Lithium Iron Phosphate, Ph.D. thesis, California Institute of Technology, Pasadena, CA, 2007.

[44] H. Dong, H. Guo, Y. He, J. Gao, W. Han, X. Lu, S. Yan, K. Yang, H. Li, D. Chen, and H. Li, Solid State Ionics 301, 133 (2017).

[45] J. Wang and X. Sun, Energy Environ. Sci. 8, 1110 (2015).

[46] See Supplemental Material at http://link.aps.org/supplemental/ 10.1103/PhysRevB.97.184405 for more information on the characterization of $\mathrm{LiFePO}_{4}$ samples and detailed structural experimental and computational results.

[47] M. Koch-Müller, E. Mugnaioli, D. Rhede, S. Speziale, U. Kolb, and R. Wirth, Am. Mineral. 99, 2405 (2009).
[48] C. M. Julien, K. Zaghib, A. Mauger, and H. Groult, Adv. Chem. Eng. Sci. 2, 321 (2012).

[49] M. Mrosko, M. Koch-Müller, C. McCammon, D. Rhede, J. R. Smyth, and R. Wirth, Contrib. Mineral. Petrol. 170, 9 (2015).

[50] K. Syassen, High Press. Res. 28, 75 (2008).

[51] M. Mrosko, M. Koch-Müller, and U. Schade, Am. Mineral. 96, 1748 (2011).

[52] H.-P. Liermann, Z. Konopkova, W. Morgenroth, K. Glazyrin, J. Bednarcik, E. E. McBride, S. Petitgirard, J. T. Delitz, M. Wendt, Y. Bican, A. Ehnes, I. Schwark, A. Rothkirch, M. Tischer, J. Heuer, H. Schulte-Schrepping, T. Kracht, and H. Franz, J. Synchrotron Radiat. 22, 908 (2015).

[53] A. P. Hammersley, S. O. Svensson, M. Hanfland, A. N. Fitch, and D. Hausermann, High Press. Res. 14, 235 (1996).

[54] B. H. Toby, J. Appl. Crystallogr. 34, 210 (2001).

[55] P. E. Blöchl, Phys. Rev. B 50, 17953 (1994).

[56] G. Kresse and D. Joubert, Phys. Rev. B 59, 1758 (1999).

[57] G. Kresse and J. Furthmüller, Comput. Mater. Sci. 6, 15 (1996).

[58] G. Kresse and J. Furthmüller, Phys. Rev. B 54, 11169 (1996).

[59] Jülich Supercomputing Centre, J. Large-Scale Res. Facilities 2 , A62 (2016).

[60] J. P. Perdew, K. Burke, and M. Ernzerhof, Phys. Rev. Lett. 77, 3865 (1996).

[61] D. M. Ceperley and B. J. Alder, Phys. Rev. Lett. 45, 566 (1980).

[62] V. I. Anisimov, J. Zaanen, and O. K. Andersen, Phys. Rev. B 44, 943 (1991).

[63] S. L. Dudarev, G. A. Botton, S. Y. Savrasov, C. J. Humphreys, and A. P. Sutton, Phys. Rev. B 57, 1505 (1998).

[64] M. T. Paques-Ledent and P. Tarte, Spectrochim. Acta, Part A 30, 673 (1974).

[65] M. T. Paques-Ledent and P. Tarte, Spectrochim. Acta, Part A 29, 1007 (1973).

[66] W. Paraguassu, P. T. C. Freire, V. Lemos, S. M. Lala, L. A. Montoro, and J. M. Rosolen, J. Raman Spectrosc. 36, 213 (2005).

[67] C. M. Burba and R. Frech, J. Electrochem. Soc. 151, A1032 (2004).

[68] A. Ait-Salah, J. Dodd, A. Mauger, R. Yazami, F. Gendron, and C. M. Julien, Z. Anorg. Allg. Chem. 632, 1598 (2006).

[69] S. Klotz, J.-C. Chervin, P. Munsch, and G. L. Marchand, J. Phys. D: Appl. Phys. 42, 075413 (2009).

[70] F. Birch, Phys. Rev. 71, 809 (1947).

[71] T. Maxisch and G. Ceder, Phys. Rev. B 73, 174112 (2006).

[72] F. Zhou, M. Cococcioni, C. A. Marianetti, D. Morgan, and G. Ceder, Phys. Rev. B 70, 235121 (2004).

[73] H. Shi, W. Luo, B. Johansson, and R. Ahuja, Phys. Rev. B 78, 155119 (2008).

[74] R. D. Shannon and C. T. Prewitt, Acta Crystallogr., Sect. B: Struct. Sci. 25, 925 (1969).

[75] J. Suntivich, K. J. May, H. A. Gasteiger, J. B. Goodenough, and Y. Shao-Horn, Science 334, 1383 (2011).

[76] D.-H. Seo, H. Gwon, S.-W. Kim, J. Kim, and K. Kang, Chem. Mater. 22, 518 (2010). 